Journal of the Optical Society of Korea, Vol. 9, No. 3, September 2005, pp. 95-98

\title{
Design and Performance Analysis of 2-Dimensional Optical CDMA Encoder/Decoder Using an Array of SSFBGs
}

\author{
Sungchul Kim* and Seoyong Shin \\ Dept. of Communications Engineering, Myong̈i University, Nam-dong, Yongin, Gyeonggi-Do 449-728, KOREA \\ Dongsun Seo \\ Dept. of Electronics Engineering, Myongji University, Nam-dong, Yongin, Gyeonggi-Do 449-728, KOREA
}

(Received August 11, 2005 : revised August 22, 2005)

\begin{abstract}
We propose a two-dimensional temporal/wavelength optical code division multiple access (OCDMA) encoder/decoder, which has an array of superstructured fiber Bragg gratings (SSFBGs). SSFBGs in an encoder/decoder have different wavelengths from each other and can be coded with the same OCDMA code or not. Simulation was performed to analyze the feasibility of the scheme.

OCIS codes : $060.0060,060.2330,230.1480$
\end{abstract}

\section{INTRODUCTION}

OCDMA is receiving substantial attention because it has attractive features such as enhanced network scalability, asynchronous operation, security, ability to support variable bit rate. In OCDMA, each user is assigned a code which is used to encode the data signal. The coded signals are transmitted and decoded at the intended receiver end by matched filtering. Hence, on the same optical bandwidth, simultaneous optical transmission can be possible. There have been many investigations of OCDMA encoding and decoding method [1-5]. Among them, FBG [6] is an attractive encoding/decoding device due to its small and compact size, low cost, good performance and suitability for mass production. Several schemes using an array of FBGs written in a sequence along a single fiber line for encoder/decoder have been proposed $[4,5]$. In these schemes, each FBG is used as a wavelength-selective reflection mirror. The spatial pasition of the fiber gratings and their associated reflection profiles can then be used to encode both temporal and spectral information onto an incident data pulse. Recently, SSFBG which uses phase coding has shown high performance in code generation and recognition. The key aspect of phase encoding allows lower inter-channel interference, and thus more simultaneous users for a given code length than conventional unipolar codes. Therefore, SSFBG provides an alternative approach to the discrete FBG array [7, 8]. Most researches using SSFBG have used a SSFBG for each user as a wavelength-coded encoder/ decoder. In this paper, we propose a novel scheme using multiple SSFBGs for each user. An array of SSFBGs written/spliced in a sequence along a single fiber line is used as an OCDMA encoder/decoder. Each SSFBG in an encoder/decoder has different wavelength and can be coded with the same OCDMA code or not. The proposed approach can increase the number of orthogonal codes, and hence, allows more simultaneous users than the system using single SSFBG. The increase of the orthogonal code can be obtained by changing the sequence of the SSFBGs and the change of the codes. In the conventional OCDMA systems using single SSFBG, the number of users is limited by the number of orthogonal codes. In the proposed system, however, the combination of the orthogonal code and the change of code sequence by changing the order of SSFBGs can give more users than that of the single SSFBG.

\section{SYSTEM DESIGN}

An array of SSFBGs is used as both temporal and wavelength coding devices. Figure 1 shows an example of an OCDMA system using the proposed scheme. There are $m$ number of users, and each user has n number of SSFBGs in the encoder. Each SSFBG has different wavelength separated by several nanometers because SSFBG has wider bandwidth than the normal FBG. The decoder is composed of SSFBGs with the reverse sequence compared to those in the encoder but with the conjugate impulse responses. Each user has multiple wavelength optical pulse sources such as mode-locked multi-wavelength laser. When the optical pulses from 


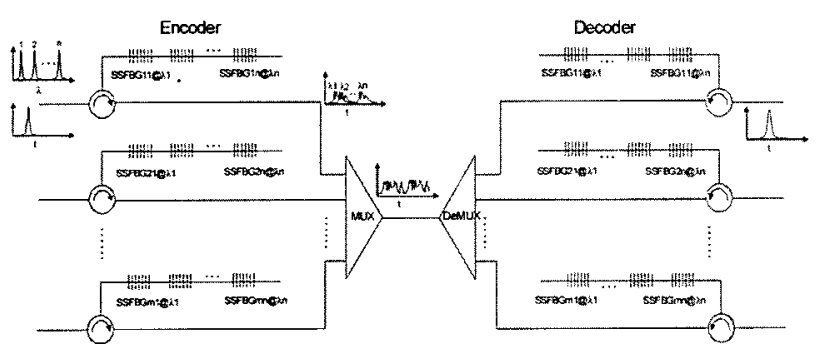

FIG. 1. Schematic diagram of the proposed temporal/ wavelength coded OCDMA encoder/decoder using an array of SSFBGs.

multi-wavelength source enter the encoder, each SSFBG reflects the pulse having the same center wavelength with the SSFBG, and generates an encoded waveform for the pulse. All of the SSFBGs in the encoder generate encoded waveforms, which are recombined into single complicated waveforms through the circulator. In the decoder, the original optical pulse is recovered through the matched filtering in SSFBGs sequenced in reverse so that the time-spread optical pulses can be transformed into a single optical pulse. In the simulation, the gold code with code length of 63 is used for SSFBG phase encoding/decoding. Figure 2 shows the reflection spectrum of a SSFBG and an array of SSFBG coded with different gold code from each other. The reflectivity of the SSFBG has to be small enough to guarantee the input optical pulse will experience the whole grating region. If the reflectivity is high, the most optical power reflected at the front end of the SSFBG and the tail area of the grating does not give any phase information to the optical signal. The small reflectivity in the SSFBG is shown in Fig. 2.

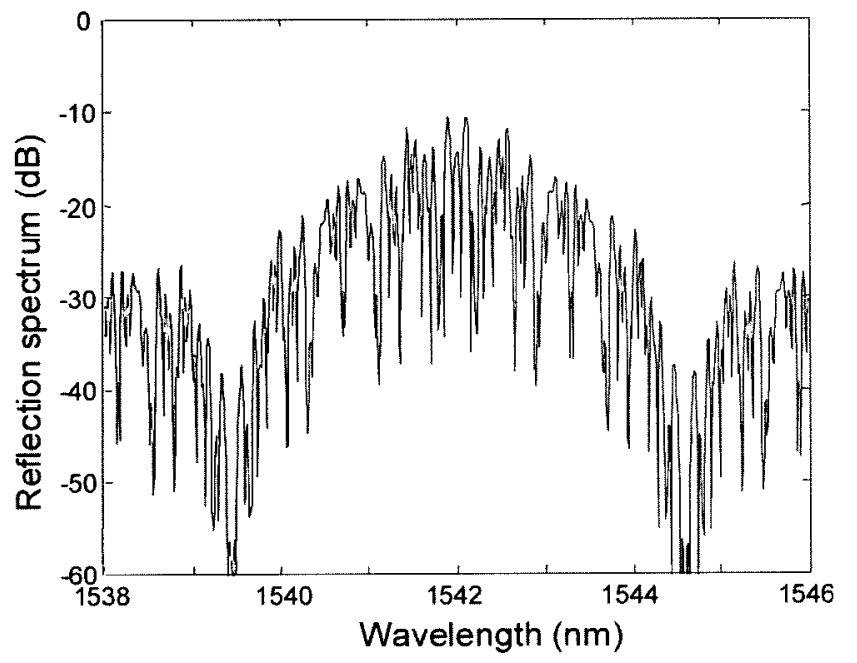

(a)

\section{SIMULATION RESULTS}

For the performance analysis of the scheme, Split-Step Fourier Method is used for optical simulation. Before verifying the performance of the proposed scheme, the orthogonal property of the optical CDMA code (gold code) used in the simulation is investigated. Figure 3 shows the encoded and decoded signals in which only a SSFBG is used to test the code. The data signal is generated by modulating the optical short pulses from a mode-locked laser through a PRBS (pseudo random bit sequence) NRZ modulator. Bit rate and optical pulse width are set to 10 Gbps and $2.5 \mathrm{ps}$, respectively. The length of SSFBG is set to $20 \mathrm{~mm}$. The data signal and the encoded signal through the encoding SSFBGs are shown in Fig. 3(a), and the comparison between the decoded signal and the original data signal is shown in Fig. 3(b). Fig 3 (c) shows the decoded signal when the decoder does not match the encoder. From the results, the gold codes we select as a phase code for fabrication of SSFBG is proper for the scheme.

To verify the scheme, the optical simulation in case of 4 users in which each encoder is composed of 4 different SSFBGs is performed. The schematic diagram is shown in Fig. 4. Each user in Fig. 4 has a multiwavelength optical pulse source, non-return-to-zero optical modulator for generating data signals, and an array of SSFBGs, which have different center wavelengths from $1542 \mathrm{~nm}$ to $1554 \mathrm{~nm}$, separated each other by $4 \mathrm{~nm}$, and hence, the optical pulse source has four wavelengths with the same center wavelength as SSFBGs. The temporal separation between SSFBGs is set to 10ps which corresponds to about $2 \mathrm{~mm}$ in fiber length. To obtain this small separation in fiber length, the SSFBGs have to be fabricated overlapped with $2 \mathrm{~mm}$ shift. This

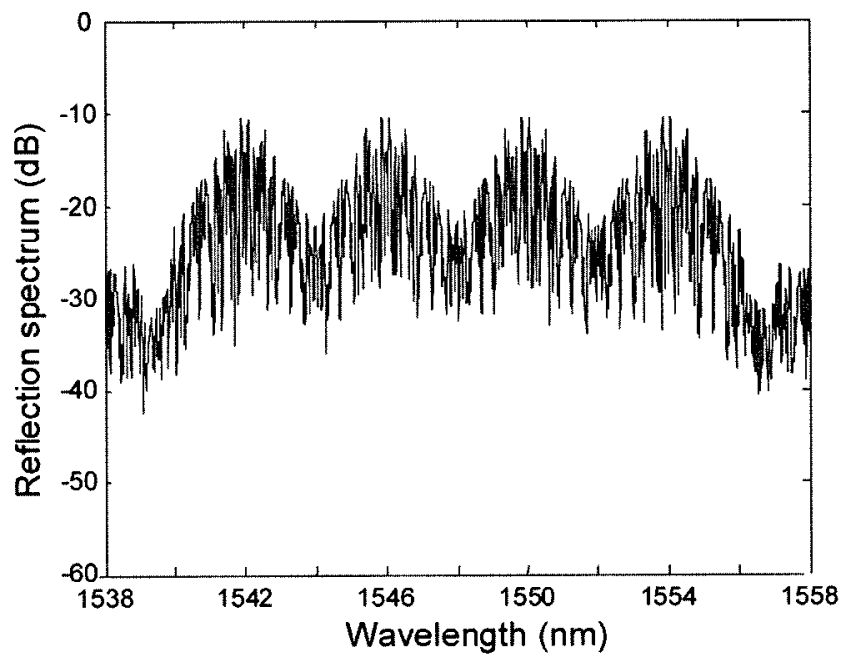

(b)

FIG. 2. Reflection spectrum of (a) a SSFBG and (b) an array of SSFBG coded with different gold codes from each other. 

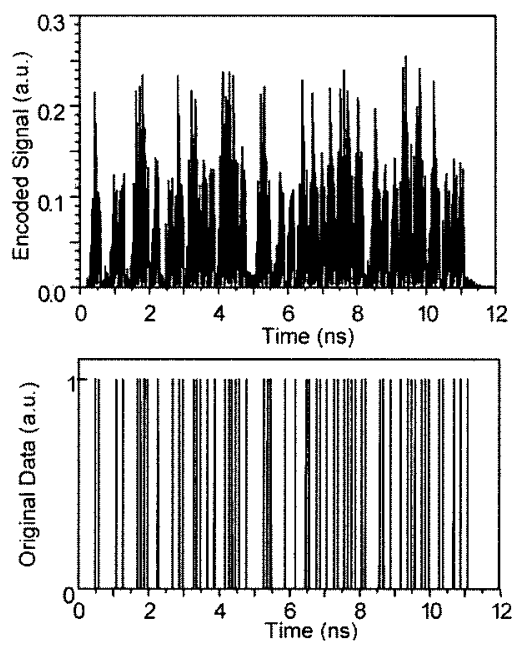

(a)
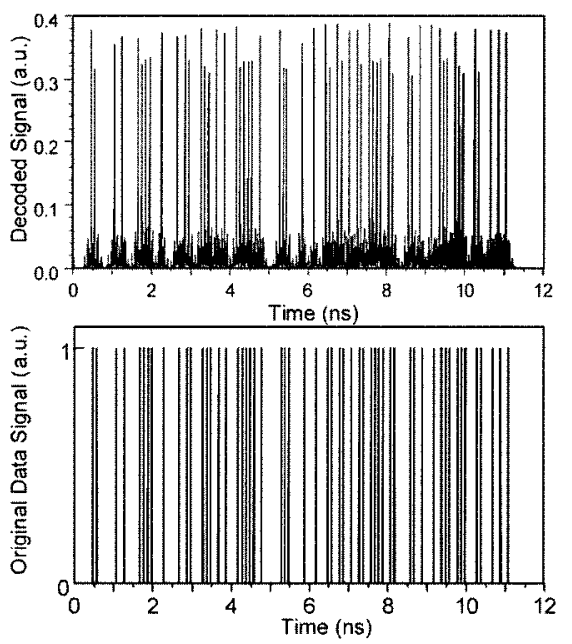

(b)

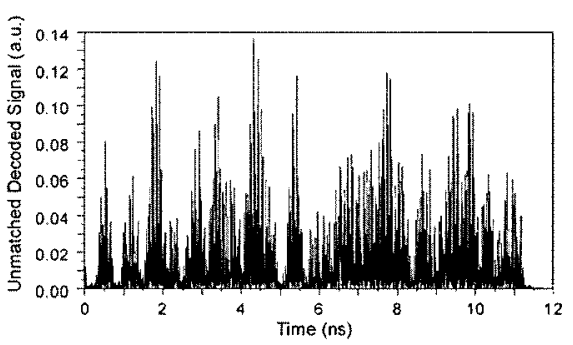

(c)

FIG. 3. Encoded and decoded signals in which only a SSFBG is used to test the code; (a) comparison between data signal and the encoded signal, (b) comparison between the decoded signal and the original data signal, (c) decoded signal when the decoder does not match the encoder.

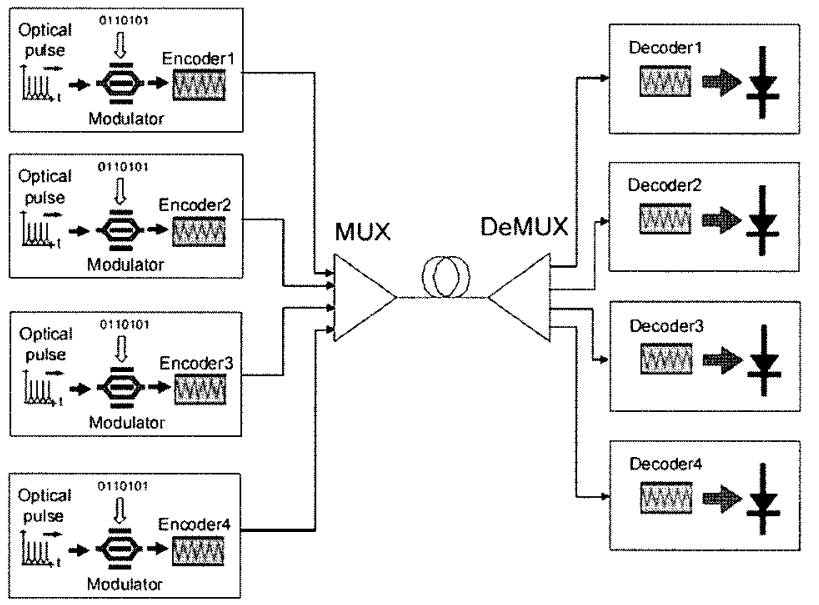

FIG. 4. Schematic Diagram in case of 4 numbers of users; each user has 4 SSFBGs each for the encoder and decoder.

does not matter because several fiber gratings can be fabricated on the same position in optical fiber if the wavelength separation between the gratings is sufficiently large. In Fig. 4, the encoder and the decoder represent 4 SSFBGs, and the optical source represents 4 different optical pulse source with center wavelength from 1542 $\mathrm{nm}$ to $1554 \mathrm{~nm}$ separated by $4 \mathrm{~nm}$. The encoded optical signals from 4 users are combined at the multiplexer (MUX), transmitted through the optical fiber, and then, split at the demultiplexer (DeMUX). In the receiver, the encoded signals from 4 different users enter the decoder. At the decoding SSFBG array, only the matched signal is properly decoded and the original optical data signal is recovered. The other signals from the unmatched encoders act as noise. In the simulation, the optical amplification is not included. Figure 5 shows the decoded signals of one of the receiver in which the encoded signals from 4 users enter into the decoding SSFBG arrays. Compared with the decoded signals of Fig. 3 (b), the background noise power was increased because the unwanted signals from the other users is not properly decoded, and then spread into the background noisy power. From the simulation results, the proposed scheme shows good performance, and the feasibility of using SSFBG arrays as an OCDMA encoder is proved.

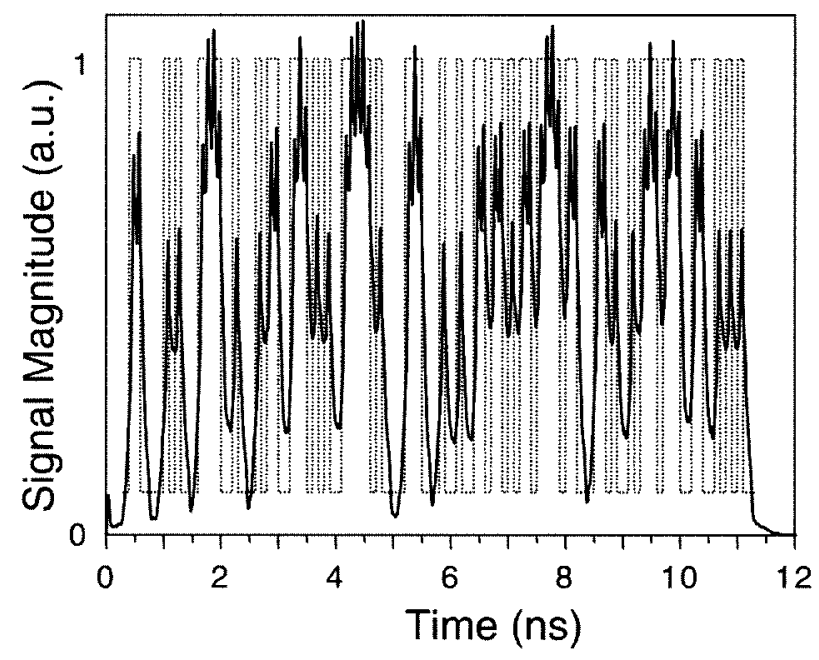

FIG. 5. Decoded signals at one of the receiver in which the encoded signals from 4 users enter into the decoding SSFBG arrays. The solid line is for the decoded signal and the dotted line is for original NRZ data. 


\section{CONCLUSION}

The 2-D (temporal/wavelength) optical CDMA encoder/ decoder using an array of SSFBGs are proposed. The feasibility of the scheme was proved by simulation of the 4-user case. The comparison between the original data and the decoded signal was shown. The Q-factor and SNR are important parameters in the performance verification of the optical system. The system performance with Q-factor and SNR, and the effects of the increase of the user to the Q-factor are still being studied and will be reported in another paper.

\section{ACKNOWLEDGEMENT}

This work was supported by Korea Research Foundation Grant (KRF-2004-042- D00140).

*Corresponding author : schkim@mju.ac.kr

\section{REFERENCES}

[1] G. J. Pendock, M. J. L. Cahill, and D. D. Sampson, "Multi-gigabit per second demonstration of photonic code-division multiplexing," Electron. Lett., vol. 31, pp. 819-820, 1995.

[2] C. F. Lam, D. T. K. Tong, M. C.Wu, and E.Yablonovitch,
"Experimental demonstration of bipolar optical CDMA system using a balanced transmitter and complementary spectral encoding," IEEE Photon. Technol. Lett., vol. 10, pp. 1504-1506, Oct. 1998.

[3] N. Wada and K. I. Kitayama, "A $10 \mathrm{~Gb} / \mathrm{s}$ optical code division multiplexing using 8-chip optical bipolar code and coherent detection," J. Lightwave Technol., vol. 17, pp. 1758-1765, Oct. 1999.

[4] H. Fathalla, L. A. Rusch, and S. LaRochelle, "Passive optical fast frequency-hop CDMA communications systems," J. Lightwave Technol, vol. 17, pp. 397-405, 1999.

[5] N. Wada, H. Sotobayashi, and K. Kitayama, "2.5Gbit/s timespread/wavelength-hop optical code division multiplexing using fiber Bragg grating with supercontinuum light source," Electron. Lett., vol. 36, pp. 815-817, Apr. $27,2000$.

[6] B. H. Lee and G. Mudhana, "Optical delay amplified by chirped fiber Bragg gratings," J. Opt. Soc. Korea, vol. 7, no. 4, pp. 224-229, 2003.

[7] P. C. The, P. Petropoulos, M. Ibsen, and D. J. Richardson, "A comparative study of the performance of seven and 63-Chip optical code-division multiple-access encoders and decoders based on superstructured fiber Bragg gratings," J. Lightwave Technol. vol. 19, no. 9, pp. 1352-1365, 2001.

[8] P. C. The, M. Ibsen, J. H. Lee, P. Petropoulos, and D. J. Richardson, "Demonstration of a four-channel WDM/ ODCMA system using 255-chip 320-Gchip/s quarternary phase coding gratings," IEEE Photon. Technol. Lett., vol. 14, no. 2, pp. 227-229, 2002. 